\title{
The 3/2 model as a stochastic volatility approximation for a large-basket price-weighted index
}

\author{
B.M. Hambly $\left.\right|^{1}$ and J. Vaicenavicius ${ }^{2}$
}

May 7, 2015

\begin{abstract}
We derive large-basket approximations of a price-weighted index whose component prices follow a single sector jump-diffusion model. As the basket size approaches infinity a suitable average converges to a Black-Scholes model driven by the common factor process. We extend this by considering the behaviour of the residual idiosyncratic noise and show that a version of the $3 / 2$ model emerges as a natural stochastic volatility model approximation. This provides a theoretical justification for its use as a model for jointly pricing index and volatility derivatives.
\end{abstract}

\section{Introduction}

A stock market index is a market statistic used to capture the performance of a portfolio of stocks traded in the market. Two of the most popular index types are price-weighted and capitalisation-weighted indices, each of which, in general, can be thought of as a weighted sum of the prices of stocks in the index basket. An example of a price-weighted index is the Dow Jones Industrial Average.

In applications an index process is usually modelled directly, assuming a specific model. Here we will take a bottom-up approach and assume a particular asset-price model for the component stocks and then consider the index as a weighted sum of these stock-price processes. The index process in this bottom up approach is naturally a very high-dimensional object. However, exploiting the averaging inherent in the weighting of the individual components, we will derive a natural stochastic volatility process for a price-weighted index in the large-basket limit.

The component assets of the index are modelled as jump diffusions driven by three noises. These correspond to a global market Brownian motion, an idiosyncratic Brownian risk factor, and an idiosyncratic shock coming from a Lévy process. One would expect that, as the basket size tends to infinity and the weights tend to zero, there should be a law of large numbers approximation; indeed, a Black-Scholes model for the index driven by the global market risk factor emerges in the limit. By considering the error in this approximation as the basket size increases we are able to produce a $3 / 2$ stochastic volatility model for the index. The version we obtain of this model has some initial

\footnotetext{
${ }^{1}$ Mathematical Institute, University of Oxford, 24-29 St Giles, Oxford OX1 3LB, UK. hambly@maths.ox.ac.uk

${ }^{2}$ Department of Mathematics, Uppsala University, Box 480, 75106 Uppsala, Sweden. juozas.vaicenavicius@math.uu.se
} 
drawbacks but suggests modifications which make the class of $3 / 2$ models seem to be natural for price-weighted indices constructed from large baskets of assets. The $3 / 2$ stochastic volatility model has recently received a lot of attention as it has other attractive features, see Carr \& Sun (2007), Drimus (2012). Along with its extensions in Baldeaux \& Badran (2013), it provides consistent modelling between index options and volatility derivatives. Thus we provide a further justification for its use.

The outline of the paper is as follows. We will begin by introducing the market model in which we work, that of asset prices following jump diffusions in a large market. We introduce the price-weighted index and other empirical moment estimators before giving our main convergence theorem describing the behaviour of the index in the large-basket limit. In Section 2 we study the resulting stochastic volatility model, showing its features, which include explosion. We then consider alternative analyses which prevent the occurrence of explosions and connect with more standard versions of the model appearing in other papers. In Section 3, we briefly discuss volatility derivatives in the model before moving to the proof of the main result in Section 4.

\subsection{The market model}

The market model that we will develop is an extension of a simple single-sector model, as considered for instance in Bush, Hambly, Haworth, Jin \& Reisinger (2011) to allow jumps in the asset-price process. Each asset will follow a jump diffusion model of the type proposed in Merton (1976), Kou (2002). In this simple model, all assets have the same constant drift, instantaneous volatility, and are correlated via a single market factor capturing global economic effects. Each asset has its own idiosyncratic noise consisting of a Brownian component and a jump component. We only put the jumps into the idiosyncratic component as such shocks will be much more common at the individual asset level, rather than the market as a whole. We note that although apparently too simplistic, as we will allow the number of assets to become infinite, a model of this type which allowed varying parameters in an infinite basket would require strong conditions on these parameters in order to avoid asymptotic arbitrage, see Kabanov \& Kramkov (1994, 1998).

Let $\left(\Omega^{n}, \mathcal{F}^{n}, \mathbb{P}^{n}\right)$ denote a probability space corresponding to a market with $n+1$ assets. We have $n$ risky assets whose prices $S_{i}(t)$ for $i=1, \ldots, n$ evolve, under a measure $\mathbb{P}^{n}$, according to the jump diffusion process

$$
\frac{\mathrm{d} S_{i}(t)}{S_{i}(t-)}=\alpha \mathrm{d} t+\sigma \rho \mathrm{d} M(t)+\sigma \sqrt{1-\rho^{2}} \mathrm{~d} W_{i}(t)+\int_{\mathbb{R} \backslash\{0\}}\left(e^{x}-1\right) N_{i}(\mathrm{~d} t, \mathrm{~d} x),
$$

where the random drivers $M, W_{i}$ are Brownian motions, $N_{i}$ is a Poisson random measure with a finite Lévy intensity measure $\nu=\lambda g$. Here $\lambda$ is the intensity of the Poisson counting process $N^{i}(t)$ and $g$ is the probability density function of the jumps $\mathcal{J}_{k}^{i}$ in the 
compound Poisson process

$$
R_{i}(t)=\int_{0}^{t} \int_{\mathbb{R} \backslash\{0\}} x N_{i}(\mathrm{~d} u, \mathrm{~d} x)=\sum_{k=1}^{N^{i}(t)} \mathcal{J}_{k}^{i}
$$

with the jumps occurring at random times $\tau_{k}^{i}$. Moreover, the processes $M, W_{1}, \ldots, W_{n}$, $R_{1}, \ldots, R_{n}$ are independent. For definitions and standard results concerning integration with respect to Poisson random measures we refer the reader to Applebaum (2009).

The coefficients in (1.1) are constants: $\alpha$ is a drift coefficient, though the total instantaneous expected return per unit time is given by $\mu=\alpha+\int_{\mathbb{R} \backslash\{0\}}\left(e^{x}-1\right) \nu(\mathrm{d} x)$ and we assume $\mu>0$; the instantaneous volatility, arising from the Brownian motion terms, is denoted by $\sigma>0$; and $\rho \in(0,1)$ is the correlation coefficient which we also assume to be strictly positive (although provided $\rho \neq 0$ we could take $\rho<0$ ). We also assume the existence of a riskless money market account paying a constant rate of interest $r$ satisfying $0<r<\mu$.

Since it is immaterial how our assets are labelled, we assume that $\left\{S_{1}(0), \ldots, S_{n}(0)\right\}$ is a family of independent identically distributed $(0, \infty)$-valued random variables with at least 8 moments. We also assume that this initial distribution is independent of $M$, $\left\{W_{i}\right\}_{1 \leq i \leq n}$, and $\left\{R_{i}\right\}_{1 \leq i \leq n}$. As we can solve the system explicitly, we can let $n \rightarrow \infty$ and our system extends to an infinite system; we write $(\Omega, \mathcal{F}, \mathbb{P})$, with an associated expectation operator $\mathbb{E}$, for the limit probability space containing the full infinite asset price model with the random initial conditions. We note that the assumptions we make on the assets could be generalised to more general jump processes and jumps in the market noise at the expense of more notation and complexity in the proofs. Here we focus on a relatively simple case to illustrate what happens.

\subsection{Price-weighted index}

Our focus will be a price-weighted market index which we define as

$$
I_{n}(t)=\frac{1}{d_{n}} \sum_{i=1}^{n} S_{i}(t)
$$

where $d_{n}$ is a constant for each $n$. We are only considering short time behaviour where the composition and weights of the index basket will remain the same. We aim to find an index approximation process $\tilde{I}_{n}$ such that $\tilde{I}_{n}(t) \approx I_{n}(t)$ for large values of $n$ and which would be driven by fewer sources of randomness. For simplicity we choose $d_{n}=n$.

Combining our model (1.1) and (1.2), the index process has dynamics

$$
I_{n}(t)=\int_{0}^{t} I_{n}(u-)\left[\left(\alpha+\beta_{1}\right) \mathrm{d} u+\rho \sigma \mathrm{d} M(u)\right]+\frac{1}{\sqrt{n}} \Pi_{n}(t),
$$


where we use the notation $\beta_{m}=\int_{\mathbb{R} \backslash\{0\}}\left(e^{m x}-1\right) \nu(\mathrm{d} x)$ for $m \in \mathbb{N}$ and

$$
\Pi_{n}(t)=\frac{\sigma \sqrt{1-\rho^{2}}}{\sqrt{n}} \sum_{i=1}^{n} \int_{0}^{t} S_{i}(u-) \mathrm{d} W_{i}(u)+\frac{1}{\sqrt{n}} \sum_{i=1}^{n} \int_{0}^{t} \int_{\mathbb{R} \backslash\{0\}} S_{i}(u-)\left(e^{x}-1\right) \tilde{N}_{i}(\mathrm{~d} u, \mathrm{~d} x),
$$

in which $\tilde{N}_{i}$ denotes the compensated Poisson random measure.

More generally, we introduce the $k$-th empirical moment process $I_{n}^{(k)}=\frac{1}{n} \sum_{i=1}^{n} S_{i}^{k}$ satisfying

$$
\begin{aligned}
\mathrm{d} I_{n}^{(k)}(t)= & \left(k \alpha+\frac{1}{2} k(k-1) \sigma^{2}+\beta_{k}\right) I_{n}^{(k)}(t-) \mathrm{d} t+k \sigma \rho I_{n}^{(k)}(t-) \mathrm{d} M(t) \\
& +k \sigma \sqrt{1-\rho^{2}} \frac{1}{n} \sum_{i=1}^{n} S_{i}(t-)^{k} \mathrm{~d} W^{i}(t)+\frac{1}{n} \sum_{i=1}^{n} S_{i}(t-)^{k} \int_{\mathbb{R} \backslash\{0\}}\left(e^{k x}-1\right) \tilde{N}_{i}(\mathrm{~d} t, \mathrm{~d} x)
\end{aligned}
$$

as well as its limit process $I^{(k)}$, which will be the solution to

$$
\mathrm{d} I^{(k)}(t)=\left(k \alpha+\frac{1}{2} k(k-1) \sigma^{2}+\beta_{k}\right) I^{(k)}(t) \mathrm{d} t+k \sigma \rho I^{(k)}(t) \mathrm{d} M(t)
$$

with the initial condition $I^{(k)}(0)=\mathbb{E}\left[S_{1}(0)^{k}\right]$ a.s. assuming the expectation is finite.

\subsection{Index approximations}

Our main mathematical result concerns weak convergence of the stochastic processes $I_{n}$ and $\Pi_{n}$ as $n \rightarrow \infty$ and provides a justification for our index approximations.

A simple observation from the structure of 1.3$)$ is that there is a law of large numbers in that, as $n \rightarrow \infty$, we will see a Black-Scholes model

$$
\mathrm{d} I^{(1)}(t)=\mu I^{(1)}(t) \mathrm{d} t+\sigma \rho I^{(1)}(t) \mathrm{d} M(t)
$$

arising in the limit.

For a more refined approximation we consider the behaviour of the error term made by the large basket approximation to a finite basket and deduce our index approximation

$$
\left\{\begin{array}{l}
\mathrm{d} I(t)=\mu I(t) \mathrm{d} t+\sigma \rho I(t) \mathrm{d} M(t)+\frac{\xi}{\sqrt{n}} \sqrt{I^{(2)}(t)} \mathrm{d} B(t), \\
\mathrm{d} I^{(2)}(t)=\left(2 \mu+\sigma^{2}+\kappa\right) I^{(2)}(t) \mathrm{d} t+2 \sigma \rho I^{(2)}(t) \mathrm{d} M(t)
\end{array}\right.
$$

where $\mu=\alpha+\beta_{1}, \gamma=\sigma^{2}\left(1-\rho^{2}\right), \kappa=\beta_{2}-2 \beta_{1}=\int_{\mathbb{R} \backslash\{0\}}\left(e^{x}-1\right)^{2} \nu(\mathrm{d} x), \xi=\sqrt{\gamma+\kappa}, M$ and $B$ are independent Brownian motions.

This is a corollary of our main theorem, which is proved in Section 4, and states the following:

Theorem 1.1. Let $\int_{\mathbb{R} \backslash\{0\}} e^{8 x} \nu(\mathrm{d} x)<\infty$ and $\mathbb{E}\left[S_{i}(0)^{8}\right]<\infty$. Then for $k=1,2$, we have

$$
I_{n}^{(k)} \Rightarrow I^{(k)}
$$


and

$$
\Pi_{n} \Rightarrow \Pi:=\int_{0} \sqrt{\xi^{2} I^{(2)}(u)} \mathrm{d} B(u) \text { as } n \rightarrow \infty,
$$

where $B$ is a standard Brownian motion independent of $M$.

The first statement (1.6) for $k=1$ yields the Black-Scholes model in the large-basket limit. For a finite-basket index approximation, we want the aggregate idiosyncratic noise contribution to be reflected as well as dependence on the basket size $n$ preserved. The statement (1.7) describes the limit behaviour of the idiosyncratic noise term $\Pi_{n}$. Thus replacing $\Pi_{n}$ with $\Pi$ in 1.3 while keeping the factor $\frac{1}{\sqrt{n}}$ in front, we obtain a refined index approximation; this can be viewed as disregarding the finite basket correction terms when approximating the correction term $\Pi_{n}$.

The initial conditions $I(0)$ and $I^{(2)}(0)$ can be viewed in two ways. As they arise from the infinite basket limits of $\frac{1}{n} \sum_{i}^{n} S_{i}(0)$ and $\frac{1}{n} \sum_{i}^{n} S_{i}(0)^{2}$ respectively, we could determine them from the basket constituents. An alternative way is to take them as inputs into the model that can be chosen to achieve the best fit during calibration.

\section{Stochastic volatility approximation}

It is time to explore the properties of the proposed index approximation (1.5).

We can write 1.5 in the form of a stochastic volatility model. Let $J=\frac{1}{n} I^{(2)} / I^{2}$. From our limit expressions for $I$ and $I^{(2)}$, a simple exercise in stochastic calculus yields the following

$$
\begin{aligned}
\mathrm{d} J= & \frac{1}{n}\left(I^{(2)} \mathrm{d} I^{-2}+I^{-2} \mathrm{~d} I^{(2)}+\mathrm{d}\left[I^{(2)}, I^{-2}\right]\right) \\
= & \frac{1}{n}\left(I^{(2)}\left(3 \sigma^{2} \rho^{2}+3 \xi^{2} J-2 \mu\right) I^{-2} \mathrm{~d} t-I^{(2)}\left(2 \sigma \rho I^{-2} \mathrm{~d} M+\frac{2 \xi \sqrt{I^{(2)}}}{\sqrt{n}} I^{-3} \mathrm{~d} B\right)\right. \\
& \left.+\left(2 \mu+\sigma^{2}+\kappa\right) I^{(2)} I^{-2} \mathrm{~d} t+2 \sigma \rho I^{(2)} I^{-2} \mathrm{~d} M-4 \sigma^{2} \rho^{2} I^{(2)} I^{-2} \mathrm{~d} t\right) \\
= & \left(\left(\sigma^{2}\left(1-\rho^{2}\right)+\kappa\right) J+3 \xi^{2} J^{2}\right) \mathrm{d} t-2 \xi J^{3 / 2} \mathrm{~d} B .
\end{aligned}
$$

Thus we have a version of the $3 / 2$ stochastic volatility model

$$
\left\{\begin{array}{l}
\mathrm{d} I(t)=\mu I(t) \mathrm{d} t+\sigma \rho I(t) \mathrm{d} M(t)+\xi I(t) \sqrt{J(t)} \mathrm{d} B(t), \\
\mathrm{d} J(t)=\xi^{2}\left(J(t)+3 J(t)^{2}\right) \mathrm{d} t-2 \xi J(t)^{3 / 2} \mathrm{~d} B(t)
\end{array}\right.
$$

with $J(0)=\frac{1}{n} I^{(2)}(0) / I(0)^{2}$. We note the influence of the basket size is now only in the initial condition for the volatility process $J$. In applications this initial condition could be determined by calibration. Also, note that as $n \rightarrow \infty$, the initial condition $J(0) \rightarrow 0$ and we see the solution $J=0$ appearing. This is consistent with our Black-Scholes limit approximation $I^{(1)}$ for the index.

Next, we give some properties of the variance process $J$. 
Proposition 2.1. (1) $Y(t)=1 / J(t)$ is a CIR process with the $S D E$

$$
\mathrm{d} Y(t)=\xi^{2}(1-Y(t)) \mathrm{d} t+2 \xi \sqrt{Y(t)} \mathrm{d} B(t) .
$$

(2) The process $X(t)=1 / \sqrt{J(t)}$ is an Ornstein-Uhlenbeck process satisfying

$$
\mathrm{d} X(t)=-\frac{1}{2} \xi^{2} X(t) \mathrm{d} t+\xi \mathrm{d} B(t)
$$

and hence the process $J(t)$ is expressed explicitly as

$$
J(t)=\left(\frac{1}{\sqrt{J(0)}} e^{-\frac{1}{2} \xi^{2} t}+\xi \int_{0}^{t} e^{\frac{1}{2} \xi^{2}(u-t)} \mathrm{d} B(u)\right)^{-2} .
$$

Proof. (1) This is Ito's formula applied to $1 / J$ with $J$ as in 2.1).

(2) Again by Ito, we see that $X$ is an Ornstein-Uhlenbeck process. Thus writing $J(t)$ in terms of the solution to the Ornstein-Uhlenbeck SDE for $X$, we have

$$
J(t)=\left(\frac{1}{\sqrt{J(0)}} e^{-\frac{1}{2} \xi^{2} t}+\xi \int_{0}^{t} e^{\frac{1}{2} \xi^{2}(u-t)} \mathrm{d} B(u)\right)^{-2} .
$$

As $X=1 / \sqrt{J}$ is an Ornstein-Uhlenbeck process, it can hit 0 and thus it is clear that the process $J$ must explode. This explosion coincides with the index crashing to 0 and is a low probability event for which we will give an explicit formula. Clearly this market collapse is a potentially serious drawback of the model. Though we note that other models with this property are still used in practice; a popular example is the Bachelier model (see Schachermayer \& Teichmann 2008).

Later we will investigate the model assuming that such an eventuality is sufficiently unlikely that we can exclude it by conditioning on non-explosion.

\subsection{Explicit characterisation of the original model}

A nice feature of the process $I$ is that it can be solved explicitly.

Theorem 2.2. The index model (2.1) is explicitly given by

$$
I(t)=I(0) e^{\left(\mu-\frac{1}{2} \sigma^{2} \rho^{2}\right) t+\sigma \rho M(t)}\left(1+\xi \sqrt{J(0)} \int_{0}^{t} e^{\frac{1}{2} \xi^{2} u} \mathrm{~d} B(u)\right) .
$$

Proof. Let $L(t)=I^{(1)}(0) / I^{(1)}(t)=e^{-\left(\mu-\frac{1}{2} \sigma^{2} \rho^{2}\right) t-\sigma \rho M(t)}$ be the process satisfying

$$
\mathrm{d} L(t)=-\left(\mu-\sigma^{2} \rho^{2}\right) L(t) \mathrm{d} t-\sigma \rho L(t) \mathrm{d} M(t) .
$$


We also write $A(t)=\sqrt{I^{(2)}(t)}=A(0) e^{\left(\mu+\frac{1}{2} \sigma^{2}\left(1-2 \rho^{2}\right)+\frac{\kappa}{2}\right) t+\sigma \rho M(t)}$. By Itô's formula,

$$
\begin{aligned}
\mathrm{d}(L(t) I(t))= & L(t) \mathrm{d} I(t)+I(t) \mathrm{d} L(t)+\mathrm{d}[I, L](t) \\
= & L(t)\left(\mu I(t) \mathrm{d} t+\sigma \rho I(t) \mathrm{d} M(t)+\frac{\xi}{\sqrt{n}} A(t) \mathrm{d} B(t)\right) \\
& +I(t)\left(-\left(\mu-\sigma^{2} \rho^{2}\right) L(t) \mathrm{d} t-\sigma \rho L(t) \mathrm{d} M(t)\right)-\sigma^{2} \rho^{2} I(t) L(t) \mathrm{d} t \\
= & \frac{\xi}{\sqrt{n}} L(t) A(t) \mathrm{d} B(t) .
\end{aligned}
$$

Hence

$$
L(t) I(t)=I(0)+\int_{0}^{t} \frac{\xi}{\sqrt{n}} L(u) A(u) \mathrm{d} B(u),
$$

and, as $L(u) A(u)=\sqrt{I^{(2)}(0)} \exp \left(\frac{1}{2} \xi^{2} u\right)$, division of both sides by $L(t)$ gives

$$
I(t)=e^{\left(\mu-\frac{1}{2} \sigma^{2} \rho^{2}\right) t+\sigma \rho M(t)}\left(I(0)+\frac{\xi}{\sqrt{n}} A(0) \int_{0}^{t} e^{\frac{1}{2} \xi^{2} u} \mathrm{~d} B(u)\right) .
$$

Rewriting in terms of $J(0)$ gives the result.

The probability of $I$ hitting zero before time $t$ can be calculated explicitly.

Proposition 2.3. Let $\tau_{0}=\inf \{t \geq 0: I(t)=0\}$ be the first time the process $I$ hits zero. Then, for $t>0$,

$$
\mathbb{P}\left(\tau_{0}<t\right)=2\left(1-\Phi\left(\frac{1}{\sqrt{J(0)\left(e^{\xi^{2} t}-1\right)}}\right)\right),
$$

where $\Phi$ is the cumulative distribution function of the standard normal distribution.

Proof. Let

$$
G(t)=\xi \sqrt{J(0)} \int_{0}^{t} e^{\frac{1}{2} \xi^{2} u} \mathrm{~d} B(u) .
$$

From the explicit solution and rewriting $G(t)$ as a time-changed Brownian motion $\tilde{B}_{[G]_{t}}$, for an independent Brownian motion $\tilde{B}$, we can write $\tau_{0}=\inf \left\{t \geq 0: \tilde{B}_{[G]_{t}}=-1\right\}$. Thus, applying the reflection principle,

$$
\begin{aligned}
\mathbb{P}\left(\tau_{0}<t\right) & =\mathbb{P}\left(\sup _{0 \leq s \leq t} \tilde{B}_{[G]_{s}}>1\right) \\
& =2 \mathbb{P}\left(\tilde{B}_{[G]_{t}}>1\right) \\
& =2\left(1-\Phi\left(\frac{1}{\sqrt{[G]_{t}}}\right)\right) .
\end{aligned}
$$

Computing the quadratic variation of $G$ gives the result.

It is easy to see that for reasonable parameter and basket size choices these probabilities are very small. 
We also see the fact that $I$ hits zero in the explosion of $J$, that is when $1 / J$, a CIR process hits zero. It is easy to check that in our case the condition on the coefficients for the CIR to hit zero is $2 \xi^{2} \leq(2 \xi)^{2}$, which holds for all $\xi$.

In the next subsections, we address the issue of the exploding stochastic variance component $J(t)$ and discuss improvements on the original index approximation model. Firstly, we will assume that explosion is sufficiently unlikely that we can condition on it not occurring. To do this, we condition on the Ornstein-Uhlenbeck process $X$ not hitting 0 . For our second version, we look more deeply into the approximation for $I^{(2)}$ and use this to show bounds on the drift and volatility of the model.

\subsection{A conditioned model with finite variance}

As $X$ hitting zero is the same event as the process $J$ exploding, the Ornstein-Uhlenbeck process conditioned not to hit 0 , denoted by $\bar{X}$, gives a model for the variance process $J$ conditioned not to explode, which we will denote by $\bar{J}$.

Lemma 2.4. The process $\bar{X}$ which is $X$ conditioned not to hit zero satisfies the SDE

$$
\mathrm{d} \bar{X}(t)=\xi^{2}\left(\frac{1}{\bar{X}(t)}-\frac{\bar{X}(t)}{2}\right) \mathrm{d} t+\xi \mathrm{d} B(t) .
$$

Proof. This comes from an application of the Doob- $h$-transform. The space-time generator $L$ of $X$ applied to a $C^{1,2}$ function $f$ is given by

$$
L f=\frac{\partial f}{\partial t}+\frac{\xi^{2}}{2} \frac{\partial^{2} f}{\partial x^{2}}-\frac{\xi^{2}}{2} x \frac{\partial f}{\partial x} .
$$

The function $h(t, x)=\exp \left(t \xi^{2} / 2\right) x$ is space-time harmonic for this process. Thus if we wish to consider $X$ conditioned not to hit zero, we need to consider the associated $h$ process. Standard calculations with the generator show that if $L^{h}$ is the $h$-transformed generator, then it is given by

$$
L^{h} f=L f+\xi^{2} \frac{1}{h} \frac{\partial h}{\partial x} \frac{\partial f}{\partial x} .
$$

Thus the SDE for the conditioned process is given by $\bar{X}$ as written above.

Remark 2.5. The Ornstein-Uhlenbeck process conditioned not to hit zero is the radial part of a three-dimensional Ornstein-Uhlenbeck process just as the Bessel process, which is Brownian motion conditioned not to hit zero, is the radial part of a three-dimensional Brownian motion.

Our new model for the index conditional on its non-collapse, $\bar{I}$, is thus

$$
\left\{\begin{array}{l}
\mathrm{d} \bar{I}(t)=\mu \bar{I}(t) \mathrm{d} t+\sigma \rho \bar{I}(t) \mathrm{d} M(t)+\xi \bar{I}(t) \sqrt{\bar{J}(t)} \mathrm{d} B(t) \\
\mathrm{d} \bar{J}(t)=\xi^{2}\left(\bar{J}(t)+\bar{J}(t)^{2}\right) \mathrm{d} t-2 \xi \bar{J}(t)^{3 / 2} \mathrm{~d} B(t)
\end{array}\right.
$$


with $\bar{J}(0)=\frac{1}{n} I^{(2)}(0) / I(0)^{2}$. Also, observe $\bar{Y}=1 / \bar{J}$ is a CIR process given by

$$
\mathrm{d} \bar{Y}(t)=\xi^{2}(3-\bar{Y}(t)) \mathrm{d} t+2 \xi \sqrt{\bar{Y}(t)} \mathrm{d} B(t) .
$$

It is easy to check that the parameters are such that almost surely this process does not hit zero.

Interestingly, we note that the instantaneous stochastic variance component $\bar{J}$ is a special case of the quadratic drift 3/2 model of Carr \& Sun (2007), which was derived therein as the unique risk-neutral variance process satisfying the stochastic volatility ratio hypothesis and the maturity independent diffusion hypothesis.

Another remark to make is that we can rewrite $(2.3)$ in the conventional stochastic volatility model form as

$$
\left\{\begin{array}{l}
\mathrm{d} \bar{I}(t)=\mu \bar{I}(t) \mathrm{d} t+\sqrt{\sigma^{2} \rho^{2}+\xi^{2} \bar{J}(t)} \bar{I}(t) \mathrm{d} W(t), \\
\mathrm{d} \bar{J}(t)=\xi^{2}\left(\bar{J}(t)+\bar{J}(t)^{2}\right) \mathrm{d} t-2 \xi \bar{J}(t)^{\frac{3}{2}} \mathrm{~d} B(t),
\end{array}\right.
$$

where

$$
W(t)=\int_{0}^{t} \frac{1}{\sqrt{\sigma^{2} \rho^{2}+\xi^{2} \bar{J}(u)}}(\sigma \rho \mathrm{d} M(u)+\xi \sqrt{\bar{J}(u)} \mathrm{d} B(u))
$$

is a Brownian motion by Lévy's characterisation. Here the Brownian motions $W(t)$ and $B(t)$ are stochastically correlated with the correlation coefficient

$$
\rho^{\prime}(t)=\frac{\xi \sqrt{\bar{J}(u)}}{\sqrt{\sigma^{2} \rho^{2}+\xi^{2} \bar{J}(u)}},
$$

coming from $\mathrm{d}[W, B](t)=\rho^{\prime}(t) \mathrm{d} t$. Thus our model $\bar{I}(t)$ falls into a class of stochastic volatility models with randomly (though endogenously) correlated Brownian drivers. Note that we have negative (even though stochastic) correlation between $\bar{I}$ and $\bar{J}$, given by $\mathrm{d}[\bar{I}, \bar{J}]=-2 \xi^{2} \bar{I} \bar{J}^{2} \mathrm{~d} t$. This is commonly seen in the market behaviour of prices and volatility (Drimus 2012). Note that in the same way, $I$ and $J$ have negative quadratic co-variation up to the explosion time of $J$.

\subsection{An alternative approach to the $3 / 2$ model}

A restrictive feature shared by $J$ as well as its conditioned version $\bar{J}$ is that there is only a single parameter available for calibration. Here we show that the large basket approach is consistent with the standard version of the $3 / 2$ stochastic volatility model with more parameters. At this point, for simplicity, we will assume that there are no jumps in the asset prices and just focus on the diffusion part. We do not prove any theorems here but just indicate that by including further corrections, the drift and volatility terms will be of the same functional form in $J$ as in our original model but with different parameters.

We begin by returning to the finite basket and perform a further analysis. In our approximation we have neglected the error that arises from the correction term in the 
empirical second moment. If we write

$$
\mathrm{d} R_{n, k}=\frac{1}{\sqrt{n}} \sum_{i=1}^{n} S_{i}^{k} \mathrm{~d} W_{i}
$$

then the first two empirical moments can be written as

$$
\begin{aligned}
\mathrm{d} I_{n} & =\mu I_{n} \mathrm{~d} t+\sigma \rho I_{n} \mathrm{~d} M+\frac{\sigma \sqrt{1-\rho^{2}}}{\sqrt{n}} \mathrm{~d} R_{n, 1} \\
\mathrm{~d} I_{n}^{(2)} & =\left(2 \mu+\sigma^{2}\right) I_{n}^{(2)} \mathrm{d} t+2 \sigma \rho I_{n}^{(2)} \mathrm{d} M+\frac{2 \sigma \sqrt{1-\rho^{2}}}{\sqrt{n}} \mathrm{~d} R_{n, 2} .
\end{aligned}
$$

As $\mathrm{d}\left[R_{n, k}\right]=I_{n}^{(2 k)} \mathrm{d} t$, we can write $\mathrm{d} R_{n, 1}=\sqrt{I_{n}^{(2)}} \mathrm{d} B_{n}$ and $\mathrm{d} R_{n, 2}=\sqrt{I_{n}^{(4)}} \mathrm{d} B_{n}^{\prime}$ for Brownian motions $B_{n}, B_{n}^{\prime}$ with

$$
\mathrm{d}\left[B_{n}, B_{n}^{\prime}\right]=\frac{I_{n}^{(3)}}{\sqrt{I_{n}^{(2)} I_{n}^{(4)}}} \mathrm{d} t
$$

A straightforward exercise with Ito's formula gives the dynamics for $J_{n}=I_{n}^{(2)} /\left(n I_{n}^{2}\right)$ as the SDE

$$
\mathrm{d} J_{n}=\sigma^{2}\left(1-\rho^{2}\right)\left(J_{n}+3 J_{n}^{2}-4 \frac{I_{n}^{(3)}}{n^{2} I_{n}^{3}}\right) \mathrm{d} t-2 \sigma \sqrt{1-\rho^{2}}\left(J_{n}^{3 / 2} \mathrm{~d} B_{n}-\left(\frac{I_{n}^{(4)}}{n^{3} I_{n}^{4}}\right)^{1 / 2} \mathrm{~d} B_{n}^{\prime}\right) .
$$

We now use elementary analytic inequalities to examine the size of the terms appearing in this SDE.

Lemma 2.6. We have the following inequalities

$$
J_{n}^{2} \leq \frac{I_{n}^{(3)}}{n^{2} I_{n}^{3}} \leq J_{n}, \quad J_{n}^{3} \leq \frac{I_{n}^{(4)}}{n^{3} I_{n}^{4}} \leq J_{n}^{2} .
$$

Proof. Firstly we note that by Hölder's inequality we have $I_{n}^{(2)} \leq \sqrt{I_{n} I_{n}^{(3)}}$, and hence

$$
J_{n}^{2} \leq \frac{I_{n}^{(3)}}{n^{2} I_{n}^{3}}
$$

Also, as

$$
J_{n}^{(k)}:=\frac{I_{n}^{(k)}}{n^{k-1} I_{n}^{k}}=\frac{\sum_{i=1}^{n} S_{i}^{k}}{\left(\sum_{i=1}^{n} S_{i}\right)^{k}},
$$

and all $S_{i}>0$, we see $J_{n}^{(k)}$ is decreasing in $k$ and hence

$$
\frac{I_{n}^{(3)}}{n^{2} I_{n}^{3}} \leq J_{n}^{(2)}=J_{n}
$$


The second inequality follows from a similar use of Hölder and monotonicity of $J_{n}^{(k)}$ in $k$.

We now show that the lower bound is the correct order in $J_{n}$.

Lemma 2.7. For all $t \geq 0$ we have

$$
\lim _{n \rightarrow \infty} \frac{I_{n}^{(3)}(t)}{n^{2} I_{n}(t)^{3}} J_{n}(t)^{-2}=\frac{\mathbb{E}\left[S_{1}(0)^{3}\right] \mathbb{E}\left[S_{1}(0)\right]}{\mathbb{E}\left[S_{1}(0)^{2}\right]^{2}} \exp \left(\sigma^{2}\left(1-\rho^{2}\right) t\right)
$$

and

$$
\lim _{n \rightarrow \infty} \frac{I_{n}^{(4)}(t)}{n^{3} I_{n}(t)^{4}} J_{n}(t)^{-3}=\frac{\mathbb{E}\left[S_{1}(0)^{4}\right] \mathbb{E}\left[S_{1}(0)\right]^{2}}{\mathbb{E}\left[S_{1}(0)^{2}\right]^{3}} \exp \left(3 \sigma^{2}\left(1-\rho^{2}\right) t\right)
$$

$\mathbb{P}$-almost surely.

Proof. As we have at least 8 moments for $S_{i}(0)$, we can just apply the strong law of large numbers to the empirical moment estimators.

Recalling our earlier analysis, if we define adapted processes $a_{n}, b_{n}$ by

$$
a_{n}(t)=\frac{I_{n}^{(3)}}{n^{2} I_{n}^{3}} J_{n}^{-2}, \quad b_{n}(t)=\frac{I_{n}^{(4)}}{n^{3} I_{n}^{4}} J_{n}^{-3},
$$

our stochastic volatility model can be written in the form

$$
\begin{aligned}
\mathrm{d} I_{n} & =\mu I_{n} \mathrm{~d} t+\sigma \rho I_{n} \mathrm{~d} M+\sigma \sqrt{1-\rho^{2}} \sqrt{J_{n}} I_{n} \mathrm{~d} B_{n} \\
\mathrm{~d} J_{n} & =\sigma^{2}\left(1-\rho^{2}\right)\left(J_{n}+\left(3-4 a_{n}(t)\right) J_{n}^{2}\right) \mathrm{d} t-2 \sigma \sqrt{1-\rho^{2}}\left(1-2 a_{n}(t)+b_{n}(t)\right)^{1 / 2} J_{n}^{3 / 2} \mathrm{~d} \tilde{B}_{n},
\end{aligned}
$$

where $\tilde{B}_{n}$ is a Brownian motion with $\mathrm{d}\left[B_{n}, \tilde{B}_{n}\right]=\left(1-a_{n}(t)\right) /\left(1-2 a_{n}(t)+b_{n}(t)\right)^{1 / 2} \mathrm{~d} t$.

Thus our approach of considering large baskets is consistent with a $3 / 2$ stochastic volatility model for a price-weighted index of the form

$$
\begin{aligned}
\mathrm{d} \hat{I} & =\hat{I}\left(\mu \mathrm{d} t+\rho \sigma \mathrm{d} M+\sigma \sqrt{1-\rho^{2}} \sqrt{\hat{J}} \mathrm{~d} W^{\prime}\right) \\
\mathrm{d} \hat{J} & =\kappa \hat{J}(\theta-\hat{J}) \mathrm{d} t+\phi \hat{J}^{3 / 2} \mathrm{~d} \tilde{W} .
\end{aligned}
$$

Here $M, W^{\prime}, \tilde{W}$ are Brownian motions, with $M$ independent of $W^{\prime}, \tilde{W}$ which are correlated. The parameter $\kappa, \theta, \phi$ are adapted processes in general. This is the type of model that has been used in recent papers such as Baldeaux \& Badran (2013), Drimus (2012) (with constant parameters).

\section{Application to volatility derivatives}

As we have obtained a stochastic volatility model for the index, we could price options on the index within the standard stochastic volatility framework. However we choose to focus on the variance process and briefly discuss how our model can price volatility derivatives in the context of our first two explicit models. 
It is straightforward to show that the model $(I, J)$ admits an equivalent martingale measure $\mathbb{Q}$ under which

$$
\left\{\begin{array}{l}
\mathrm{d} I(t)=r I(t) \mathrm{d} t+\sigma \rho I(t) \mathrm{d} M^{\mathbb{Q}}(t)+\xi I(t) \sqrt{J(t)} \mathrm{d} B^{\mathbb{Q}}(t), \\
\mathrm{d} J(t)=\xi^{2}\left(J(t)+3 J(t)^{2}\right) \mathrm{d} t-2 \xi J(t)^{3 / 2} \mathrm{~d} B^{\mathbb{Q}}(t),
\end{array}\right.
$$

where $M^{\mathbb{Q}}, B^{\mathbb{Q}}$ are independent $\mathbb{Q}$-Brownian motions. Likewise for the model $(\bar{I}, \bar{J})$ with non-exploding variance, there is an equivalent martingale measure $\overline{\mathbb{Q}}$ under which the model takes the form

$$
\left\{\begin{array}{l}
\mathrm{d} \bar{I}(t)=r \bar{I}(t) \mathrm{d} t+\sigma \rho \bar{I}(t) \mathrm{d} M^{\overline{\mathbb{Q}}}(t)+\xi \bar{I}(t) \sqrt{\bar{J}(t)} \mathrm{d} B^{\overline{\mathbb{Q}}}(t), \\
\mathrm{d} \bar{J}(t)=\xi^{2}\left(\bar{J}(t)+\bar{J}(t)^{2}\right) \mathrm{d} t-2 \xi \bar{J}(t)^{3 / 2} \mathrm{~d} B^{\overline{\mathbb{Q}}}(t)
\end{array}\right.
$$

with $M^{\overline{\mathbb{Q}}}, B^{\overline{\mathbb{Q}}}$ being independent $\overline{\mathbb{Q}}$-Brownian motions.

Remark 3.1. (1) The price of volatility swaps or calls on realised variance will be infinite in the exploding model. The prices of puts on realised variance will always be finite.

(2) We note that in performing a Monte Carlo simulation over a one year time interval for $10^{6}$ paths and reasonable parameter values, one is unlikely to see the explosion of $J$.

We will mainly focus on the non-exploding model and define the instantaneous variance $V(t)=\sigma^{2} \rho^{2}+\xi^{2} \bar{J}(t)$ and the realised variance $Q(t)$ by

$$
Q(t)=\int_{0}^{t} \mathrm{~d}[\log \bar{I}](s)=\sigma^{2} \rho^{2} t+\xi^{2} \int_{0}^{t} \bar{J}(s) \mathrm{d} s .
$$

We note that the CIR process $\bar{Y}$ has negative moments up to $3 / 2$, so that $\mathbb{E}_{\overline{\mathbb{Q}}} \bar{J}^{k}<$ $\infty$ for all $k<3 / 2$. For the original exploding model, the associated CIR process $Y$ has negative moments up to $1 / 2$ in that $\mathbb{E}_{\mathbb{Q}} J^{k}<\infty$ for $k<1 / 2$. See the paper by Hurd \& Kuznetsov (2008) for such properties of the CIR process. We remark that the results in Hurd \& Kuznetsov (2008) also allow us to derive a formula for the Laplace transform of the realized variance in this model. We let $v_{\theta}=-1 / 4+\sqrt{1+8 \theta} / 4$ and $\zeta=\frac{1}{2}\left(1-\exp \left(-\xi^{2} T\right)\right)^{-1}$. Then for $\theta \geq-1 / 8$ we have

$$
\begin{aligned}
\mathbb{E}_{\overline{\mathbb{Q}}}[\exp (-\theta Q(T))] & =e^{-\theta \sigma^{2} \rho^{2} T} \mathbb{E}_{\overline{\mathbb{Q}}}\left[\exp \left(-\theta \xi^{2} \int_{0}^{T} \bar{Y}_{t}^{-1} \mathrm{~d} t\right)\right] \\
& =e^{-\theta \sigma^{2} \rho^{2} T-\xi^{2} v_{\theta} T} \bar{J}(0)^{-v_{\theta}} \zeta^{v_{\theta}} \frac{\Gamma\left(\frac{3}{2}+v_{\theta}\right)}{\Gamma\left(\frac{3}{2}+2 v_{\theta}\right)}{ }_{1} F_{1}\left(v_{\theta}, \frac{3}{2}+2 v_{\theta} ;-\frac{\zeta}{\bar{J}(0)} e^{-\xi^{2} T}\right),
\end{aligned}
$$

where ${ }_{1} F_{1}$ is a confluent hypergeometric function and $\Gamma$ is the gamma function.

The pricing of variance swaps is straightforward within the model for $\bar{J}$. The time 0 price, $v_{0}$, of the swap over the period $[0, T]$ is given by

$$
v_{0}=\mathbb{E}_{\overline{\mathbb{Q}}}[Q(T)]=\sigma^{2} \rho^{2} T+\xi^{2} \int_{0}^{T} \mathbb{E}_{\overline{\mathbb{Q}}}[\bar{J}(s)] \mathrm{d} s .
$$


As the inverse of the CIR has moments of order up to 3/2, we have by Hurd \& Kuznetsov (2008) that

$$
\begin{aligned}
\mathbb{E}_{\overline{\mathbb{Q}}}[\bar{J}(s)] & =\mathbb{E}_{\overline{\mathbb{Q}}}\left[\bar{Y}_{s}^{-1}\right] \\
& =\frac{1}{1-\exp \left(-\xi^{2} s\right)}{ }_{1} F_{1}\left(1, \frac{3}{2},-\frac{\exp \left(-\xi^{2} s\right)}{2 \bar{J}(0)\left(1-\exp \left(-\xi^{2} s\right)\right)}\right) .
\end{aligned}
$$

Substituting this back into the variance swap price, rewriting the confluent hypergeometric function in standard integral form, and then changing variables we have that

$$
v_{0}=\mathbb{E}_{\overline{\mathbb{Q}}}[Q(T)]=\sigma^{2} \rho^{2} T+\int_{z_{T}}^{\infty} \int_{0}^{1} \frac{e^{-z u}}{2 z(1-u)^{1 / 2}} \mathrm{~d} u \mathrm{~d} z
$$

where $z_{T}=\frac{\exp \left(-\xi^{2} T\right)}{2 \bar{J}(0)\left(1-\exp \left(-\xi^{2} T\right)\right)}$. Unfortunately, there does not seem to be an explicit formula in this case, unlike say the standard Heston model. The prices of volatility swaps and options on variance or volatility with the model would have to be computed numerically.

\section{Weak convergence theorems}

This final section is dedicated to the proof of the main convergence result, Theorem 1.1 . The two constituent parts of the theorem are proved separately as Theorem 4.6 and Corollary 4.8 .

\subsection{Convergence preliminaries}

Before we proceed with the proofs, some known results that will be used in due course are gathered below for completeness. Firstly, we state a diffusion approximation theorem, which will be our main tool in proving convergence to a diffusion process. The following version appears as Theorem 4.1 on page 354 of Ethier \& Kurtz (1989).

Theorem 4.1 (Rebolledo 1979). Let $a=\left(\left(a_{i j}\right)\right)$ be a continuous, symmetric, nonnegative definite, $d \times d$ matrix-valued function on $\mathbb{R}^{d}$ and let $b: \mathbb{R}^{d} \rightarrow \mathbb{R}^{d}$ be continuous. Let

$$
A(a, b)=\left\{\left(f, G f \equiv \frac{1}{2} \sum a_{i j} \partial_{i} \partial_{j} f+\sum b_{i} \partial_{i} f\right): f \in C_{c}^{\infty}\left(\mathbb{R}^{d}\right)\right\},
$$

where $C_{c}^{\infty}\left(\mathbb{R}^{d}\right)$ denotes the class of compactly supported infinitely differentiable functions on $\mathbb{R}^{d}$, and suppose that the $C_{\mathbb{R}^{d}}[0, \infty)$ martingale problem for $A$ is well-posed.

For $n \in\{1,2, \ldots\}$, let $X_{n}$ and $B_{n}$ be processes with sample paths in $D_{\mathbb{R}^{d}}[0, \infty)$, and let $A_{n}=\left(\left(A_{n}^{i j}\right)\right)$ be a symmetric $d \times d$ matrix-valued process such that $A_{n}^{i j}$ has sample paths in $D_{\mathbb{R}^{d}}[0, \infty)$ and $A_{n}(t)-A_{n}(s)$ is non-negative definite for $t>s \geq 0$. Set $\mathcal{F}_{t}^{n}=$ $\sigma\left(X_{n}(s), B_{n}(s), A_{n}(s): s \leq t\right)$.

Let $\tau_{n}^{r}=\inf \left\{t \geq 0:\left|X_{n}(t)\right| \geq r\right.$ or $\left.\left|X_{n}(t-)\right| \geq r\right\}$, and suppose that

$$
M_{n} \equiv X_{n}-B_{n}
$$


and

$$
M_{n}^{i} M_{n}^{j}-A_{n}^{i j}, \quad i, j=1, \ldots, d,
$$

are $\left\{\mathcal{F}_{t}^{n}\right\}$-local martingales, and that for each $r>0, T>0$, and $i, j=1, \ldots, d$,

$$
\begin{array}{r}
\lim _{n \rightarrow \infty} \mathbb{E}\left[\sup _{t \leq T \wedge \tau_{n}^{r}}\left|X_{n}(t)-X_{n}(t-)\right|^{2}\right]=0, \\
\lim _{n \rightarrow \infty} \mathbb{E}\left[\sup _{t \leq T \wedge \tau_{n}^{r}}\left|B_{n}(t)-B_{n}(t-)\right|^{2}\right]=0, \\
\lim _{n \rightarrow \infty} \mathbb{E}\left[\sup _{t \leq T \wedge \tau_{n}^{r}}\left|A_{n}^{i j}(t)-A_{n}^{i j}(t-)\right|=0,\right. \\
\sup _{t \leq T \wedge \tau_{n}^{r}}\left|B_{n}^{i}(t)-\int_{0}^{t} b_{i}\left(X_{n}(s)\right) \mathrm{d} s\right| \rightarrow 0 \text { in probability } \\
\sup _{t \leq T \wedge \tau_{n}^{r}}\left|A_{n}^{i j}(t)-\int_{0}^{t} a_{i j}\left(X_{n}(s)\right) \mathrm{d} s\right| \rightarrow 0 \text { in probability. }
\end{array}
$$

Suppose that $\mathbb{P} \circ X_{n}(0)^{-1} \Rightarrow \eta \in \mathcal{P}\left(\mathbb{R}^{d}\right)$. Then $\left\{X_{n}\right\}$ converges in distribution to the solution of the martingale problem for $(A, \eta)$.

The following elementary inequality will be handy for checking a condition of the diffusion approximation theorem.

Theorem 4.2 (Arnold \& Groeneveld 1979). Let $X_{1}, \ldots, X_{n}$ be jointly distributed with common expectation $\mu$ and variance $\sigma^{2}$. Then

$$
\mathbb{E}\left[\max _{1 \leq i \leq n} X_{i}\right] \leq \mu+\sigma \sqrt{n-1}
$$

\subsection{The limit Black-Scholes model}

In this part, we will establish the limiting behaviour of the index process $I_{n}$. Indeed, we will prove convergence of the $k$-th empirical moment process $I_{n}^{(k)}=\frac{1}{n} \sum_{i=1}^{n} S_{i}^{k}$ to a limiting diffusion process $I^{(k)}$ as $n \rightarrow \infty$. We begin by proving a few simple lemmas. The conditions given in the following lemmas are merely sufficient and by no means the best possible as our aim is to explore an idea rather than derive results of maximum generality.

Lemma 4.3. Let $m \in \mathbb{N}$ and suppose that $\mathbb{E}\left[S_{i}(0)^{m}\right]<\infty$ and $\int_{\mathbb{R} \backslash\{0\}} e^{m x} \nu(\mathrm{d} x)<\infty$. Then for all positive $k \leq m$ and all $t \geq 0, \mathbb{E}\left[S_{i}(t)^{k}\right]=\mathbb{E}\left[S_{1}(t)^{k}\right]<\infty$.

Proof. It is enough to show that $\mathbb{E}\left[S_{1}(t)^{m}\right]<\infty$ and this is standard as we have an explicit form for $S_{1}(t)$. 
Lemma 4.4. Suppose that $\mathbb{E}\left[S_{i}(t)^{2 k}\right]<\infty$ for all $t \geq 0$. Then for any $T>0$ and $k \in \mathbb{N}$,

$$
\mathbb{E}\left[\left(\sup _{t \leq T} \frac{1}{\sqrt{n}} \int_{0}^{t} I_{n}^{(k)}(u) \mathrm{d} u\right)^{2}\right] \rightarrow 0 \text { as } n \rightarrow \infty .
$$

Proof. Since $I_{n}^{(k)}(u)$ is always positive, we have that $\int_{0}^{t} \frac{1}{\sqrt{n}} I_{n}^{(k)}(u) \mathrm{d} u$ is increasing in $t$, and an application of Cauchy-Schwarz gives

$$
\begin{aligned}
\mathbb{E}\left[\left(\sup _{0 \leq t \leq T} \int_{0}^{t} \frac{1}{\sqrt{n}} I_{n}^{(k)}(u) \mathrm{d} u\right)^{2}\right] & \leq \mathbb{E}\left[\left(\int_{0}^{T} \frac{1}{\sqrt{n}} I_{n}^{(k)}(u) \mathrm{d} u\right)^{2}\right] \\
& \leq \frac{T}{n} \mathbb{E}\left[\int_{0}^{T} I_{n}^{(k)}(u)^{2} \mathrm{~d} u\right] .
\end{aligned}
$$

Using the Cauchy-Schwarz inequality again we have, for all $u \geq 0$,

$$
\mathbb{E}\left[I_{n}^{(k)}(u)^{2}\right] \mathrm{d} u \leq \frac{1}{n^{2}} \mathbb{E}\left[n \sum_{i=1}^{n} S_{i}(u)^{2 k}\right]=\mathbb{E} I_{n}^{(2 k)}(u)=\mathbb{E} S_{1}(u)^{2 k}<\infty,
$$

and, as the bound is positive and increasing, by Fubini's theorem, $\mathbb{E}\left[\int_{0}^{T} I_{n}^{(k)}(u)^{2} \mathrm{~d} u\right]<\infty$. Thus, letting $n \rightarrow \infty$ in 4.9 , gives the result.

Lemma 4.5. Let $T>0, k \in \mathbb{N}$ and suppose that $\mathbb{E}\left[S_{i}(t)^{2 k}\right]<\infty$ for all $t \geq 0$. Then for any $p>\frac{1}{2}$

$$
\frac{1}{n^{p}} \mathbb{E}\left[\sup _{1 \leq i \leq n} \sup _{t \leq T} S_{i}(t)^{k}\right] \rightarrow 0 \quad \text { as } n \rightarrow \infty .
$$

Proof. Let us write $Z_{i}:=\sup _{t \leq T} S_{i}(t)^{k}$. By Doob's submartingale inequality,

$$
\mathbb{E}\left[Z_{i}^{2}\right]=\mathbb{E}\left[\sup _{t \leq T} S_{i}(t)^{2 k}\right] \leq \frac{2 k}{2 k-1} \mathbb{E}\left[S_{i}(T)^{2 k}\right]<\infty,
$$

so both mean and variance of $Z_{i}$ exist and we call them $\mu_{i}$ and $\sigma_{i}$ respectively. Also, note that for any $t \leq 0, S_{1}(t), \ldots, S_{n}(t)$ are identically distributed, therefore, $Z_{1}, \ldots, Z_{n}$ are also identically distributed with $\mu_{i}=\mu, \sigma_{i}=\sigma$ for all $i \in\{1, \ldots, n\}$. Now, by Theorem 4.2 , we obtain

$$
\frac{1}{n^{p}} \mathbb{E}\left[\max _{1 \leq i \leq n} Z_{i}\right] \leq \frac{1}{n^{p}}(\mu+\sigma \sqrt{n-1}) \rightarrow 0 \quad \text { as } n \rightarrow \infty .
$$

Now, we state and prove a result about the behaviour of $I_{n}^{(k)}$ as $n \rightarrow \infty$. The notation is as in Section 1.2. 
Theorem 4.6. Let $k \in \mathbb{N}$ and suppose that $\mathbb{E}\left[S_{i}(0)^{4 k}\right]<\infty$ and $\int_{\mathbb{R} \backslash\{0\}} e^{4 k x} \nu(\mathrm{d} x)<\infty$. Then $I_{n}^{(k)} \Rightarrow I^{(k)}$ as $n \rightarrow \infty$, where the process $I^{(k)}$ is given by

$$
\left\{\begin{array}{l}
\mathrm{d} I^{(k)}(t)=\left(k \alpha+\frac{1}{2} k(k-1) \sigma^{2}+\beta_{k}\right) I^{(k)}(t) \mathrm{d} t+k \sigma \rho I^{(k)}(t) \mathrm{d} M(t), \\
I^{(k)}(0)=\mathbb{E}\left[S_{1}(0)^{k}\right] .
\end{array}\right.
$$

Proof. We first note that the martingale problem for $I^{(k)}$ is well posed as it is a geometric Brownian motion. We will apply the diffusion approximation theorem (Theorem 4.1) to show that $X_{n}=I_{n}^{(k)}$ converges to $I^{(k)}$.

Applying Itô's formula we obtain the dynamics of $I_{n}^{(k)}$ as

$$
\begin{aligned}
\mathrm{d} I_{n}^{(k)}(t)=(k \alpha & \left.+\frac{1}{2} k(k-1) \sigma^{2}+\beta_{k}\right) I_{n}^{(k)}(t-) \mathrm{d} t+k \sigma \rho I_{n}^{(k)}(t-) \mathrm{d} M(t) \\
& +k \sigma \sqrt{1-\rho^{2}} \frac{1}{n} \sum_{i=1}^{n} S_{i}(t-)^{k} \mathrm{~d} W^{i}(t) \\
& +\frac{1}{n} \sum_{i=1}^{n} S_{i}(t-)^{k} \int_{\mathbb{R} \backslash\{0\}}\left(e^{k x}-1\right) \tilde{N}_{i}(\mathrm{~d} t, \mathrm{~d} x) .
\end{aligned}
$$

Letting

$$
B_{n}(t)=\int_{0}^{t}\left(k \alpha+\frac{1}{2} k(k-1) \sigma^{2}+\beta_{k}\right) I_{n}^{(k)}(u) \mathrm{d} u,
$$

clearly, $M_{n}=X_{n}-B_{n}$ is a local martingale. Furthermore, letting

$$
\begin{aligned}
A_{n}(t)= & {\left[M_{n}\right](t) } \\
= & \int_{0}^{t} k^{2} \sigma^{2} \rho^{2} I_{n}^{(k)}(u)^{2}+k^{2} \sigma^{2}\left(1-\rho^{2}\right) \frac{1}{n} I_{n}^{(2 k)}(u) \mathrm{d} u \\
& +\frac{1}{n^{2}} \sum_{i=1}^{n} \int_{0}^{t} S_{i}(u-)^{2 k} \int_{\mathbb{R} \backslash\{0\}}\left(e^{k x}-1\right)^{2} N_{i}(\mathrm{~d} u, \mathrm{~d} x),
\end{aligned}
$$

by the Doob-Meyer decomposition (c.f. Ethier \& Kurtz 1989 p. 79 ), $M_{n}^{2}-A_{n}$ is a local martingale.

Now, the conditions 4.4 -4.8 need to be checked. Since the jumps occur at distinct times almost surely,

$$
\begin{aligned}
\mathbb{E}\left[\sup _{t \leq T}\left|X_{n}(t)-X_{n}(t-)\right|^{2}\right] & =\mathbb{E}\left[\sup _{1 \leq i \leq n} \sup _{t \leq T}\left(\frac{1}{n}\left(S_{i}^{k}(t)-S_{i}^{k}(t-)\right)\right)^{2}\right] \\
& \leq \frac{1}{n^{2}} \mathbb{E}\left[\sup _{1 \leq i \leq n} \sup _{t \leq T} S_{i}(t)^{2 k}\right]
\end{aligned}
$$

Likewise,

$$
\mathbb{E}\left[\sup _{t \leq T}\left|A_{n}(t)-A_{n}(t-)\right|\right] \leq \frac{1}{n^{2}} \mathbb{E}\left[\sup _{1 \leq i \leq n} \sup _{t \leq T} S_{i}(t)^{2 k}\right]
$$


Thus by Lemma 4.5 , both $\mathbb{E}\left[\sup _{t \leq T}\left|X_{n}(t)-X_{n}(t-)\right|^{2}\right]$ and $\mathbb{E}\left[\sup _{t \leq T}\left|A_{n}(t)-A_{n}(t-)\right|\right]$ converge to 0 as $n \rightarrow \infty$, proving the conditions (4.4) and (4.6). The jump condition (4.5) is trivially satisfied because the $B_{n}$ are continuous in t. Also, the condition (4.7) trivially holds because

$$
B_{n}(t)-\int_{0}^{t} b\left(X_{n}(u)\right) \mathrm{d} u=0 \quad \text { for all } t \geq 0 .
$$

To establish the condition 4.8, let us denote

$$
\begin{gathered}
G_{n}(t):=\int_{0}^{t} k^{2} \sigma^{2}\left(1-\rho^{2}\right) \frac{1}{n} I_{n}^{(2 k)}(u) \mathrm{d} u \\
H_{n}(t):=\frac{1}{n^{2}} \sum_{i=1}^{n} \int_{0}^{t} \int_{\mathbb{R} \backslash\{0\}} S_{i}(u-)^{2 k}\left(e^{k x}-1\right)^{2} N_{i}(\mathrm{~d} u, \mathrm{~d} x) .
\end{gathered}
$$

Consider

$$
\begin{aligned}
\mathbb{E}\left[\sup _{t \leq T}\left|A_{n}(t)-\int_{0}^{t} a\left(X_{n}(u)\right) \mathrm{d} u\right|^{2}\right] & \leq \mathbb{E}\left[\left(\sup _{t \leq T}\left|G_{n}(t)\right|+\sup _{t \leq T}\left|H_{n}(t)\right|\right)^{2}\right] \\
& \leq 2 \mathbb{E}\left[\sup _{t \leq T}\left|G_{n}(t)\right|^{2}\right]+2 \mathbb{E}\left[\sup _{t \leq T}\left|H_{n}(t)\right|^{2}\right] .
\end{aligned}
$$

For 4.8 to hold, it is sufficient to show that $\mathbb{E}\left[\sup _{t \leq T}\left|G_{n}(t)\right|^{2}\right]$ and $\mathbb{E}\left[\sup _{t \leq T}\left|H_{n}(t)\right|^{2}\right]$ converge to 0 as $n \rightarrow \infty$. The term

$$
\mathbb{E}\left[\sup _{t \leq T}\left|G_{n}(t)\right|^{2}\right]=k^{4} \sigma^{4}\left(1-\rho^{2}\right)^{2} \frac{1}{n} \mathbb{E}\left[\sup _{t \leq T}\left|\int_{0}^{t} \frac{1}{\sqrt{n}} I_{n}^{(2 k)}(u) \mathrm{d} u\right|^{2}\right]
$$


converges to 0 as $n \rightarrow \infty$ by Lemma 4.4. Also,

$$
\begin{aligned}
\mathbb{E}\left[\sup _{t \leq T}\left|H_{n}(t)\right|^{2}\right] & =\mathbb{E}\left[H_{n}(T)^{2}\right] \\
& =\frac{1}{n^{4}} \mathbb{E}\left[\left(\sum_{i=1}^{n} \sum_{0 \leq u \leq T}\left(S_{i}(u)^{k}-S_{i}(u-)^{k}\right)^{2}\right)^{2}\right] \\
& \leq \frac{1}{n^{3}} \mathbb{E}\left[\sum_{i=1}^{n}\left(\sum_{0 \leq u \leq T}\left(S_{i}(u)^{k}-S_{i}(u-)^{k}\right)^{2}\right)^{2}\right] \\
& \leq \frac{1}{n^{2}} \mathbb{E}\left[N^{1}(T) \sum_{j=1}^{N^{1}(T)} S_{1}\left(\tau_{j}^{1}-\right)^{4 k}\left(e^{k \mathcal{J}_{j}^{1}}-1\right)^{4}\right] \\
& =\frac{1}{n^{2}} \sum_{N=1}^{\infty} \mathbb{E}\left[N \sum_{j=1}^{N} S_{1}\left(\tau_{j}^{1}-\right)^{4 k}\left(e^{k \mathcal{J}_{j}^{1}}-1\right)^{4}\right] \mathbb{P}\left(N^{1}(T)=N\right) \\
& \leq \frac{1}{n^{2}} \sum_{N=1}^{\infty} N^{2}\left(\sup _{t \leq T} \mathbb{E}\left[S_{1}(t)^{4 k}\right]\right) \mathbb{E}\left[\left(e^{k \mathcal{J}_{j}^{1}}-1\right)^{4}\right] \mathbb{P}\left(N^{1}(T)=N\right) \\
& =\frac{1}{n^{2}}\left(\sup _{t \leq T} \mathbb{E}\left[S_{1}(t)^{4 k}\right]\right) \mathbb{E}\left[\left(e^{k \mathcal{J}_{j}^{1}}-1\right)^{4}\right] \mathbb{E}\left[N^{1}(T)^{2}\right] \\
& \rightarrow 0 \quad \operatorname{as} n \rightarrow \infty .
\end{aligned}
$$

In the above, the first two inequalities hold by Cauchy-Schwartz, the third one by independence of $S_{1}\left(\tau_{j}^{1}-\right)$ and $\mathcal{J}_{j}^{1}$.

Lastly, noting that by the law of large numbers, $\mathbb{P} \circ I_{n}^{(k)}(0)^{-1} \Rightarrow \delta_{\mathbb{E}\left[S_{1}(0)^{k}\right]}$, we have finished checking the conditions of Theorem 4.1 and so the proof is completed.

\subsection{Approximation of the idiosyncratic noise}

In this subsection, we will be concerned with the large $n$ limit behaviour of the scaled idiosyncratic noise term $\Pi_{n}$ in (1.4). Alternatively, one can view the term as $\Pi_{n}(t)=$ $\sqrt{n}\left(I_{n}(t)-I^{(1)}(t)\right)$, which can be thought of as a scaled fluctuation of $I_{n}(t)$ around the first approximation $I^{(1)}(t)$. Scaled by $\sqrt{n}$, the idiosyncratic noise no longer vanishes as in the first approximation, but weakly converges to a non-trivial process $\Pi$. The result comes as a corollary of the following theorem. The notation is as in Section 1.2 .

Theorem 4.7. Let

$$
X_{n}(t):=\left(\begin{array}{c}
\Pi_{n}(t) \\
\xi^{2} I_{n}^{(2)}(t)
\end{array}\right)
$$

and

$$
X(t):=\left(\begin{array}{c}
\int_{0}^{t} \xi \sqrt{I^{(2)}(u)} \mathrm{d} B(u) \\
\xi^{2} I^{(2)}(t)
\end{array}\right),
$$

where $B$ is a standard Brownian motion independent of $M$. Also, suppose that $\mathbb{E}\left[S_{i}(0)^{8}\right]<\infty$ 
and $\int_{\mathbb{R} \backslash\{0\}} e^{8 x} \nu(\mathrm{d} x)<\infty$.

Then $X_{n} \Rightarrow X$ as $n \rightarrow \infty$.

As weak convergence of vectors implies weak convergence of their components, we have the following immediate corollary.

Corollary 4.8. Under the conditions of Theorem 4.7 and with the same notation, $\Pi_{n} \Rightarrow \int_{0}^{\cdot} \xi \sqrt{I^{(2)}(u)} \mathrm{d} B(u)$ as $n \rightarrow \infty$.

In order to prove Theorem 4.7 we again use Theorem 4.1 to show that $X_{n}$ converges weakly to the solution of a well-posed martingale problem solved by $X$. This martingale problem has the generator

$$
\begin{gathered}
b(x, y)=\left(\begin{array}{c}
0 \\
\left(2 \alpha+\sigma^{2}+\beta_{2}\right) y
\end{array}\right), \\
a(x, y)=\left(\begin{array}{cc}
|y| & 0 \\
0 & 4 \sigma^{2} \rho^{2} y^{2}
\end{array}\right),
\end{gathered}
$$

and the initial condition $\eta=\delta_{X(0)}$, where $X(0):=\left(0, \xi^{2} \mathbb{E}\left[S_{1}(0)^{2}\right]\right)^{T}$. By the StroockVaradhan theorem (see p. 170 of Rogers \& Williams 2000), the martingale problem is well-posed. Also, note that by the law of large numbers, so $\mathbb{P} \circ X_{n}(0)^{-1} \Rightarrow \delta_{X(0)}$.

We define

$$
B_{n}(t)=\left(\begin{array}{c}
0 \\
\xi^{2} \int_{0}^{t}\left(2 \alpha+\sigma^{2}+\beta_{2}\right) I_{n}^{(2)}(u) \mathrm{d} u
\end{array}\right) .
$$

so that $M_{n}=X_{n}-B_{n}$ has no drift part and is a local martingale. Then by the DoobMeyer decomposition (c.f. Ethier \& Kurtz 1989 p. 79), we choose

$$
A_{n}^{i j}(t)=\left[M_{n}^{i}, M_{n}^{j}\right](t)=G_{n}^{i j}(t)+H_{n}^{i, j}(t) \quad(1 \leq i, j \leq 2),
$$

where

$$
\begin{aligned}
& G_{n}^{11}(t)=\gamma \int_{0}^{t} I_{n}^{(2)}(u) \mathrm{d} u \\
& G_{n}^{12}(t)=G_{n}^{21}(t)=\frac{2 \xi^{2} \gamma}{\sqrt{n}} \int_{0}^{t} I_{n}^{(3)}(u) \mathrm{d} u \\
& G_{n}^{22}(t)=\xi^{4}\left(4 \sigma^{2} \rho^{2} \int_{0}^{t}\left(I_{n}^{(2)}(u)\right)^{2} \mathrm{~d} u+\frac{4 \gamma}{n} \int_{0}^{t} I_{n}^{(4)}(u) \mathrm{d} u\right), \\
& H_{n}^{11}(t)=\frac{1}{n} \sum_{i=1}^{n} \int_{0}^{t} \int_{\mathbb{R} \backslash\{0\}} S_{i}(u-)^{2}\left(e^{x}-1\right)^{2} N_{i}(\mathrm{~d} u, \mathrm{~d} x), \\
& H_{n}^{12}(t)=H_{n}^{21}(t)=\xi^{2} n^{-\frac{3}{2}} \sum_{i=1}^{n} \int_{0}^{t} \int_{\mathbb{R} \backslash\{0\}} S_{i}(u-)^{3}\left(e^{2 x}-1\right)\left(e^{x}-1\right) N_{i}(\mathrm{~d} u, \mathrm{~d} x), \\
& H_{n}^{22}(t)=\frac{\xi^{4}}{n^{2}} \sum_{i=1}^{n} \int_{0}^{t} \int_{\mathbb{R} \backslash\{0\}} S_{i}(u-)^{4}\left(e^{2 x}-1\right)^{2} N_{i}(\mathrm{~d} u, \mathrm{~d} x) .
\end{aligned}
$$


The conditions of the diffusion approximation theorem are checked in the subsequent lemmas.

Lemma 4.9. For any $t>s \geq 0, A_{n}(t)-A_{n}(s)$ is non-negative definite.

Proof. It is enough to show that $G_{n}(t)-G_{n}(s)$ and $H_{n}(t)-H_{n}(s)$ are non-negative definite. By the Cauchy-Schwarz inequality applied twice,

$$
\begin{aligned}
\left(\int_{s}^{t} \sum_{i=1}^{n} S_{i}^{2}(u) \mathrm{d} u\right)\left(\int_{s}^{t} \sum_{i=1}^{n} S_{i}^{4}(u) \mathrm{d} u\right) & \geq\left(\int_{s}^{t}\left(\sum_{i=1}^{n} S_{i}^{2}(u)\right)^{\frac{1}{2}}\left(\sum_{i=1}^{n} S_{i}^{4}(u)\right)^{\frac{1}{2}} \mathrm{~d} u\right)^{2} \\
& \geq\left(\int_{s}^{t} \sum_{i=1}^{n} S_{i}(u)^{3} \mathrm{~d} u\right)^{2},
\end{aligned}
$$

which implies

$$
\left(G_{n}^{11}(t)-G_{n}^{11}(s)\right)\left(G_{n}^{22}(t)-G_{n}^{22}(s)\right) \geq\left(G_{n}^{12}(t)-G_{n}^{12}(s)\right)^{2} .
$$

Also, by another application of the Cauchy-Schwarz inequality,

$$
\left(\sum_{i=1}^{n} \sum_{s \leq u \leq t}\left(\Delta S_{i}(u)\right)^{2}\right)\left(\sum_{i=1}^{n} \sum_{s \leq u \leq t}\left(\Delta S_{i}^{2}(u)\right)^{2}\right) \geq\left(\sum_{i=1}^{n} \sum_{s \leq u \leq t} \Delta S_{i}(u) \Delta S_{i}^{2}(u)\right)^{2},
$$

where we use the jump notation $\Delta Y(t)=Y(t)-Y(t-)$ for a real-valued càdlàg process $Y$. This implies that

$$
\left(H_{n}^{11}(t)-H_{n}^{11}(s)\right)\left(H_{n}^{22}(t)-H_{n}^{22}(s)\right) \geq\left(H_{n}^{12}(t)-H_{n}^{12}(s)\right)^{2} .
$$

Hence both $G_{n}(t)-G_{n}(s)$ and $H_{n}(t)-H_{n}(s)$ are non-negative definite.

Lemma 4.10. Conditions (4.5) and (4.7) are satisfied.

Proof. Since $B_{n}(t)$ is continuous, then the jump condition 4.5 trivially holds. Next, the condition (4.7) is also trivially satisfied because $B_{n}(t)-\int_{0}^{t} b\left(X_{n}(u)\right) \mathrm{d} u=0$.

Lemma 4.11. Suppose that $\mathbb{E}\left[S_{i}(t)^{8}\right]<\infty$ for all $t \geq 0$. Then the jump conditions (4.4) and (4.6) are satisfied. 
Proof. We begin by considering

$$
\begin{aligned}
\mathbb{E} & {\left[\sup _{t \leq T}\left|X_{n}(t)-X_{n}(t-)\right|^{2}\right] } \\
& =\mathbb{E}\left[\sup _{t \leq T}\left(\left(\frac{1}{\sqrt{n}} \sum_{i=1}^{n}\left(S_{i}(t)-S_{i}(t-)\right)\right)^{2}+\left(\frac{\xi^{2}}{n} \sum_{i=1}^{n}\left(S_{i}(t)^{2}-S_{i}(t-)^{2}\right)\right)^{2}\right)\right] \\
& =\frac{1}{n} \mathbb{E}\left[\sup _{t \leq T} \sum_{i=1}^{n}\left(S_{i}(t)-S_{i}(t-)\right)^{2}\right]+\frac{\xi^{4}}{n^{2}} \mathbb{E}\left[\sup _{t \leq T} \sum_{i=1}^{n}\left(S_{i}(t)^{2}-S_{i}(t-)^{2}\right)^{2}\right] \\
& \leq \frac{1}{n} \mathbb{E}\left[\sup _{1 \leq i \leq n} \sup _{t \leq T} S_{i}(t)^{2}\right]+\frac{\xi^{4}}{n^{2}} \mathbb{E}\left[\sup _{1 \leq i \leq n} \sup _{t \leq T} S_{i}(t)^{4}\right],
\end{aligned}
$$

where we repeatedly have used the fact that no two jumps occur at the same time almost surely. Similarly,

$$
\begin{aligned}
\mathbb{E}\left[\sup _{t \leq T}\left|A_{n}^{11}(t)-A_{n}^{11}(t-)\right|\right] & =\mathbb{E}\left[\sup _{t \leq T} \frac{1}{n} \sum_{i=1}^{n}\left(S_{i}(t)-S_{i}(t-)\right)^{2}\right] \\
& \leq \frac{1}{n} \mathbb{E}\left[\sup _{1 \leq i \leq n} \sup _{t \leq T} S_{i}(t)^{2}\right]
\end{aligned}
$$

and

$$
\begin{aligned}
& \mathbb{E}\left[\sup _{t \leq T}\left|A_{n}^{12}(t)-A_{n}^{12}(t-)\right|\right] \\
& \quad=\mathbb{E}\left[\sup _{t \leq T} \xi^{2} n^{-\frac{3}{2}} \sum_{i=1}^{n}\left|S_{i}(t)^{2}-S_{i}(t-)^{2}\right|\left|S_{i}(t)-S_{i}(t-)\right|\right] \\
& =\xi^{2} n^{-\frac{3}{2}} \mathbb{E}\left[\sup _{1 \leq i \leq n} \sup _{t \leq T}\left|S_{i}(t)^{2}-S_{i}(t-)^{2}\right|\left|S_{i}(t)-S_{i}(t-)\right|\right] \\
& \leq \xi^{2} n^{-\frac{3}{2}} \mathbb{E}\left[\sup _{1 \leq i \leq n} \sup _{t \leq T} S_{i}(t)^{3}\right],
\end{aligned}
$$

and

$$
\begin{aligned}
\mathbb{E}\left[\sup _{t \leq T}\left|A_{n}^{22}(t)-A_{n}^{22}(t-)\right|\right] & =\mathbb{E}\left[\sup _{t \leq T} \frac{\xi^{4}}{n^{2}} \sum_{i=1}^{n}\left(S_{i}(t)^{2}-S_{i}(t-)^{2}\right)^{2}\right] \\
& \leq \frac{\xi^{4}}{n^{2}} \mathbb{E}\left[\sup _{1 \leq i \leq n} \sup _{t \leq T} S_{i}(t)^{4}\right]
\end{aligned}
$$

Now, we observe that by Lemma 4.5, every expectation above converges to 0 as $n \rightarrow \infty$, therefore the conditions (4.4) and (4.6) are satisfied.

Lemma 4.12. Suppose that $\mathbb{E}\left[S_{i}(0)^{8}\right]<\infty$ and $\int_{\mathbb{R} \backslash\{0\}} e^{8 x} \nu(\mathrm{d} x)<\infty$. Then the condition (4.8) is satisfied. 
Proof. We need to verify all three cases of the condition 4.8.

(i) Case: $i=j=1$.

$$
U_{n}(t):=A_{n}^{11}(t)-\int_{0}^{t} a_{11}\left(X_{n}(u)\right) \mathrm{d} u=\frac{1}{n} \sum_{i=1}^{n} \int_{0}^{t} \int_{\mathbb{R} \backslash\{0\}}\left(e^{x}-1\right)^{2} S_{i}(u-)^{2} \tilde{N}(\mathrm{~d} u, \mathrm{~d} x) .
$$

Let us fix $T>0$ and note that $U_{n}^{T}:=U_{n}(\cdot \wedge T)$ is a martingale, so $\left|U_{n}^{T}\right|$ is a positive submartingale and we can apply Doob's submartingale inequality giving

$$
\mathbb{E}\left[\sup _{t \leq T}\left|U_{n}(t)\right|^{2}\right]=\mathbb{E}\left[\sup _{t \leq T}\left|U_{n}^{T}(t)\right|^{2}\right] \leq 4 \mathbb{E}\left[\left|U_{n}^{T}(T)\right|^{2}\right]=4 \mathbb{E}\left[U_{n}(T)^{2}\right] .
$$

Let

$$
Y_{i}(T):=\int_{0}^{T} \int_{\mathbb{R} \backslash\{0\}} S_{i}(u-)^{2}\left(e^{x}-1\right)^{2} \tilde{N}_{i}(\mathrm{~d} u, \mathrm{~d} x) .
$$

Then

$$
\mathbb{E}\left[U_{n}(T)^{2}\right]=\frac{1}{n^{2}} \sum_{i=1}^{n} \mathbb{E}\left[Y_{i}(T)^{2}\right]+\frac{1}{n^{2}} \sum_{\substack{i, k=1 \\ i \neq k}}^{n} \mathbb{E}\left[Y_{i}(T) Y_{k}(T)\right]
$$

Now, suppose that $i \neq k$ and consider the second term in the expression above. As our underlying probability space can be considered as the product space $\left(\Omega_{\left\{S_{i}(0)\right\}_{1}^{n}} \times \Omega_{M} \times \prod_{i=1}^{n} \Omega_{W_{i}} \times \prod_{i=1}^{n} \Omega_{R_{i}}, \prod_{i=1}^{n} \mathbb{P}^{S_{i}(0)} \times \mathbb{P}^{M} \times \prod_{i=1}^{n} \mathbb{P}^{W_{i}} \times \prod_{i=1}^{n} \mathbb{P}^{R_{i}}\right)$ with a natural sigma-algebra, $Y_{i}(T)$ and $Y_{k}(T)$ are independent conditional on the market factor $M$.

Since $Y_{i}(\cdot \wedge T)$ conditioned on any sample path of $M$ is a martingale,

$$
\mathbb{E}\left[Y_{i}(T) \mid \sigma(M)\right]=\mathbb{E}\left[Y_{i}(0) \mid \sigma(M)\right]=0 .
$$

Hence, the second term in 4.12 is 0 . By the Itô isometry for integrals with respect to a compensated Poisson random measure,

$$
\begin{aligned}
\mathbb{E}\left[Y_{i}(T)^{2}\right] & =\mathbb{E}\left[\left(\int_{0}^{T} \int_{\mathbb{R} \backslash\{0\}} S_{i}(u-)^{2}\left(e^{x}-1\right)^{2} \tilde{N}_{i}(\mathrm{~d} u, \mathrm{~d} x)\right)^{2}\right] \\
& =\int_{0}^{T} \int_{\mathbb{R} \backslash\{0\}} \mathbb{E}\left[S_{i}(u)^{4}\left(e^{x}-1\right)^{4}\right] \nu(\mathrm{d} x) \mathrm{d} u \\
& =\int_{\mathbb{R} \backslash\{0\}}\left(e^{x}-1\right)^{4} \nu(\mathrm{d} x) \int_{0}^{T} \mathbb{E}\left[S_{i}(u)^{4}\right] \mathrm{d} u,
\end{aligned}
$$

which is bounded above by some constant $C$ because the fourth moment of $S_{i}$ exists and the jump intensity measure density $\nu$ is such that $\int_{\mathbb{R} \backslash\{0\}}\left(e^{x}-1\right)^{4} \nu(\mathrm{d} x)$ is finite. 
Consequently, from 4.12 we obtain that

$$
\mathbb{E}\left[U_{n}(T)^{2}\right]=\frac{1}{n^{2}} n \mathbb{E}\left[Y_{i}(T)^{2}\right]<\frac{1}{n} C \rightarrow 0 \quad \text { as } n \rightarrow \infty,
$$

and thus using 4.11 we can conclude that $\mathbb{E}\left[\sup _{t \leq T}\left|U_{n}(t)\right|^{2}\right] \rightarrow 0$ as $n \rightarrow \infty$.

(ii) Case: $i=1, j=2$.

Recall

$$
A_{n}^{12}(t)-\int_{0}^{t} a_{12}\left(X_{n}(u)\right) \mathrm{d} u=G_{n}^{12}(t)+H_{n}^{12}(t) .
$$

Now, consider

$$
\begin{aligned}
\mathbb{E}\left[\sup _{t \leq T} H_{n}^{12}(t)^{2}\right] & =\mathbb{E}\left[H_{n}^{12}(T)^{2}\right] \\
& \leq \frac{\xi^{4}}{n^{3}} \mathbb{E}\left[n \sum_{i=1}^{n}\left(\int_{0}^{T} \int_{\mathbb{R} \backslash\{0\}} S_{i}(u-)^{3}\left(e^{2 x}-1\right)\left(e^{x}-1\right) N_{i}(\mathrm{~d} u, \mathrm{~d} x)\right)^{2}\right] \\
& =\frac{\xi^{4}}{n} \mathbb{E}\left[\left(\sum_{j=1}^{N^{1}(T)} S_{1}\left(\tau_{j}^{1}-\right)^{3}\left(e^{2 \mathcal{J}_{j}^{1}}-1\right)\left(e^{\mathcal{J}_{j}^{1}}-1\right)\right)^{2}\right] \\
& \leq \frac{\xi^{4}}{n} \mathbb{E}\left[N^{1}(T) \sum_{j=1}^{N^{1}(T)} S_{1}\left(\tau_{j}^{1}-\right)^{6}\left(e^{2 \mathcal{J}_{j}^{1}}-1\right)^{2}\left(e^{\mathcal{J}_{j}^{1}}-1\right)^{2}\right] \\
& =\frac{\xi^{4}}{n} \sum_{N=1}^{\infty} \mathbb{E}\left[N \sum_{j=1}^{N} S_{1}\left(\tau_{j}^{1}-\right)^{6}\left(e^{2 \mathcal{J}_{j}^{1}}-1\right)^{2}\left(e^{\mathcal{J}_{j}^{1}}-1\right)^{2}\right] \mathbb{P}\left(N^{1}(T)=N\right) \\
& \leq \frac{\xi^{4}}{n} \sum_{N=1}^{\infty} N^{2}\left(\sup _{t \leq T} \mathbb{E}\left[S_{1}(t)^{6}\right]\right) \mathbb{E}\left[\left(e^{2 \mathcal{J}_{1}^{1}}-1\right)^{2}\left(e^{\mathcal{J}_{1}^{1}}-1\right)^{2}\right] \mathbb{P}\left(N^{1}(T)=N\right) \\
& =\frac{1}{n} \xi^{4}\left(\sup _{t \leq T} \mathbb{E}\left[S_{1}(t)^{6}\right]\right) \mathbb{E}\left[\left(e^{2 \mathcal{J}_{1}^{1}}-1\right)^{2}\left(e^{\mathcal{J}_{1}^{1}}-1\right)^{2}\right] \mathbb{E}\left[N^{1}(T)^{2}\right] \\
& \rightarrow 0 \quad \text { as } n \rightarrow \infty
\end{aligned}
$$

In the above, the first two inequalities hold by Cauchy-Schwarz, the third inequality follows from independence of $S_{1}\left(\tau_{j}^{1}-\right)$ and $\mathcal{J}_{j}^{1}$.

Also, by Lemma 4.4,

$$
\sup _{t \leq T}\left|G_{n}^{12}(t)\right| \rightarrow 0 \quad \text { in } L^{2} \text { as } n \rightarrow \infty
$$

consequently,

$$
\mathbb{E}\left[\sup _{t \leq T}\left|G_{n}^{12}(t)+H_{n}^{12}(t)\right|^{2}\right] \leq 2 \mathbb{E}\left[\sup _{t \leq T}\left|G_{n}^{12}(t)\right|^{2}\right]+2 \mathbb{E}\left[\sup _{t \leq T}\left|H_{n}^{12}(t)\right|^{2}\right] \rightarrow 0
$$


as $n \rightarrow \infty$.

(iii) Case: $i=j=2$.

$$
A_{n}^{22}(t)-\int_{0}^{t} a_{22}\left(X_{n}(u)\right) \mathrm{d} u=\xi^{4}\left(U_{n}(t)+V_{n}(t)\right),
$$

where

$$
\begin{gathered}
U_{n}(t)=\frac{4 \gamma}{n} \int_{0}^{t} I_{n}^{(4)}(u) \mathrm{d} u \\
V_{n}(t)=\frac{1}{n^{2}} \sum_{i=1}^{n} \int_{0}^{t} \int_{\mathbb{R} \backslash\{0\}} S_{i}(u-)^{4}\left(e^{2 x}-1\right)^{2} N_{i}(\mathrm{~d} u, \mathrm{~d} x) .
\end{gathered}
$$

Notice that by an analogous argument as in (ii) above,

$$
\mathbb{E}\left[\sup _{t \leq T}\left|V_{n}(t)\right|^{2}\right] \rightarrow 0 \quad \text { as } n \rightarrow \infty,
$$

the only difference being that for this we use $\mathbb{E}\left[S_{1}(t)^{8}\right]<\infty$ and $\mathbb{E}\left[e^{8 \mathcal{J}_{1}^{1}}\right]<\infty$. Also, by applying Lemma 4.4 to $U_{n}(t)$, we obtain that

$$
\mathbb{E}\left[\sup _{t \leq T}\left|U_{n}(t)\right|^{2}\right] \rightarrow 0 \quad \text { as } n \rightarrow \infty \text {. }
$$

Consequently,

$$
\mathbb{E}\left[\sup _{t \leq T}\left|U_{n}(t)+V_{n}(t)\right|^{2}\right] \leq 2 \mathbb{E}\left[\sup _{t \leq T}\left|U_{n}(t)\right|^{2}\right]+2 \mathbb{E}\left[\sup _{t \leq T}\left|V_{n}(t)\right|^{2}\right] \rightarrow 0 \quad \text { as } n \rightarrow \infty .
$$

Thus the condition 4.8 is satisfied.

Proof of Theorem 4.7. As all the conditions of Theorem 4.1 have been checked above, we can conclude that $X_{n}$ converges weakly to the solution of the martingale problem for $X$, which completes the proof of Theorem 4.7 .

Remark 4.13. If we trace back through the proof above, we see that similar arguments yield

$$
\left(\begin{array}{c}
\frac{1}{\sqrt{n}} \sum_{i=1}^{n} \int_{0}^{\cdot} S_{i}(u-) \mathrm{d} W_{i}(u) \\
I_{n}^{(2)}
\end{array}\right) \Rightarrow\left(\begin{array}{c}
\int_{0}^{\cdot} \sqrt{I^{(2)}(u)} \mathrm{d} B_{1}(u) \\
I^{(2)}
\end{array}\right)
$$

and

$$
\left(\begin{array}{c}
\frac{1}{\sqrt{n}} \sum_{i=1}^{n} \int_{0} \int_{\mathbb{R} \backslash\{0\}} S_{i}(u-)\left(e^{x}-1\right) \tilde{N}_{i}(\mathrm{~d} u, \mathrm{~d} x) \\
\kappa I_{n}^{(2)}
\end{array}\right) \Rightarrow\left(\begin{array}{c}
\int_{0}^{\cdot} \sqrt{\kappa I^{(2)}(u)} \mathrm{d} B_{2}(u) \\
\kappa I^{(2)}
\end{array}\right),
$$

where $B_{1}, B_{2}$ are Brownian motions independent of $M$. It is worth noting that although 
the idiosyncratic terms are of different nature, one continuous, another pure jump, they both weakly converge to the same process up to a scaling constant.

\section{References}

[1] D. Applebaum, Lévy Processes and Stochastic Calculus, Cambridge studies in advanced mathematics 116 (Second edition, Cambridge University Press, 2009).

[2] B. C. Arnold and R. A. Groeneveld, Bounds on Expectations of Linear Systematic Statistics Based on Dependent Samples, Ann. Statist., 7, (1979), 220-223.

[3] J. Baldeaux and A. Badran, Consistent modelling of VIX and equity derivatives using a 3/2 plus jumps model, Appl. Math. Finance, 21, (2013), 299-312.

[4] N. Bush, B. M. Hambly, H. Haworth, L. Jin, and C. Reisinger, Stochastic evolution equations in portfolio credit modelling, SIAM J. Financial Math., 2, (2011), 627-664.

[5] P. Carr and J. Sun, A new approach for option pricing under stochastic volatility, Rev. Deriv. Research 10, (2007), 87-150.

[6] J. C. Cox, J. .E. Ingersoll, S. A. Ross, A Theory of the Term Structure of Interest Rates, Econometrica, 53, (1985), 385-408.

[7] S. N. Ethier and T. G. Kurtz, Markov Processes - Characterisation and Convergence (Wiley, 1989).

[8] G.G. Drimus, Options on realized variance by transform methods: A non-affine stochastic volatility model, Quant. Finance, 12, (2012), 1679-1694.

[9] S. L. Heston, A Closed-Form Solution for Options with Stochastic Volatility with Applications to Bond and Currency Options, Rev. Fin. Studies, 6, (1993), 327-343.

[10] T.R. Hurd and A. Kuznetsov, Explicit formulas for Laplace transforms of stochastic integrals, Markov Process. Related Fields, 14, (2008), 277-290.

[11] S. G. Kou, A Jump-Diffusion Model for Option Pricing, Manag. Sci., 48, (2002), 1086-1101.

[12] Yu. M. Kabanov and D. O. Kramkov, Large financial markets: asymptotic arbitrage and contiguity. Theory Probab. Appl., 39, (1995), 182-187.

[13] Yu. M. Kabanov and D. O. Kramkov, Asymptotic arbitrage in large financial markets. Finance Stoch., 2, (1998), 143-172.

[14] R. C. Merton, Option pricing when underlying stock returns are discontinuous, $J$. Fin. Economics, 3, (1976), 125-144.

[15] R. Rebolledo, La méthod des martingales appliquée à l'étude de la convergence en loi de processus, Bull. Soc. Math. France Mem., 62, (1979). 
[16] L. C. G. Rogers and D. Williams, Diffusions, Markov Processes and Martingales, Volume II (Second edition, Cambridge University Press, 2000).

[17] W. Schachermayer, J. Teichmann, How close are the Option Pricing Formulas of Bachelier and Black-Merton-Scholes?, Math. Finance, 18, (2008), 155-170. 